\title{
Congenital Heart Defects in Children Born to Women with Systemic Lupus Erythematosus in Dubai, a Nested Case-Control Study pf Ajch Patient Records between 2015-2017
}

\author{
Mahmoud Al Soufi and Rashed Khalid Rowaiee* \\ Department of Medicine, Al Jalila Children's Speciality Hospital-Heart Center, UAE
}

*Corresponding author: Rashed Khalid Rowaiee, Al Jalila Children's Speciality Hospital-

Received Date: December 19, 2018

Heart Center, Mohammed Bin Rashid University of Medicine and Health Sciences, UAE.

Published Date: January 17, 2019

\begin{abstract}
Background and aim: It has been speculated by some studies that the exposure to systemic lupus erythematosus (SLE) in utero may lead to the development of congenital heart defects (CHD) in the growing fetus. The evidence we have on the truth of this correlation is weak and needs to be elucidated. We determined to study whether children who are born to women with SLE have an increased risk of CHD when compared to children born to women without SLE.

Methods and results: Data was collected retrospectively from patient records at Al Jalila Children's Hospital (AJCH) between 2015 and 2017. The patient records were of mothers with SLE aged between 20 and 40 matched with mothers without SLE of the same age group. We then followed them to see whether or not they had children with CHD. The diagnosis of CHD was made on a fetal echocardiogram during the gestational period. In total, we identified 122 women who were eligible in the study. We ran chi-squared tests and found 36 women had SLE compared to 86 who did not have SLE. Of the 36 women with SLE, only 5 had children with CHD. Of the non-SLE women, 35 women had children with CHD. From the risk estimate, we found that women with SLE are 4.2 times more likely to have children with CHD when compared to non-SLE women (OR 4.2, 95\%CI 0.083-0.664). This was confirmed in Fisher's Exact test with a P-value of 0.005, indicating significant difference between the two groups. We then carried out a logistical regression analysis for age of fetus, age of mother and nationality. Results indicate that none of these variables play a significant role in influencing the main correlation in the study.
\end{abstract}

Conclusion: Compared to children who are born to mothers without SLE, children born to mothers with SLE have an increased risk of CHD.

Keywords: Systemic lupus erythematosus; Congenital heart defect; Pregnancy; Epidemiology

\section{Key Messages}

\section{What is already known about this subject?}

- It is known that systemic lupus erythematosus can act as a risk factor for developing congenital heart defects in the growing fetus.

\section{What does this study add?}

- Fills in the gap in the literature where some papers suggest no or weak association between SLE and CHD.
- $\quad$ Elucidates the correlation between SLE and CHD.

How might this impact on public health/clinical/ pedagogical practice?

- $\quad$ Aids the physician in making more informed and evidence based advice to mothers with SLE willing to start a family.

- Helps in better management in the healthcare system by recommending more rigorous care plans to the mother. 


\section{Introduction}

\section{Background and rationale}

Systemic Lupus Erythematosus (SLE) is a rare autoimmune disorder which involves multiple organs and is characterized by autoantibodies production which cause injury by binding the antibodies to various organ tissues and forming immune complex depositions. It is known that some antibodies can cross the placental barrier and circulate in the fetus' circulation, causing damage to multiple organs which may retard the growth of the fetus. This disease predominantly affects women of childbearing age with a ratio of 8:1 compared to men [1]. Extensive research has been carried out on the effects of common risk factors, such as diabetes and smoking, on the growing fetus. However, there has been minimal research regarding SLE's consequences on the development of a healthy fetus due to the rarity of the disease.

Congenital Heart defects (CHD) are among the most common types of birth defects seen, accounting for 8 of 1,000 births each year [2]. In utero exposures to maternal chronic illnesses and medication are thought to manifest an important role in the etiology of CHD. A study has found that children born to mothers with various systemic connective tissue diseases, including SLE, have a 3-fold increase in the risk of CHD compared to controls [3]. It has been suggested by previous studies 4 that there is a correlation between SLE and CHD in the fetus. Hence, due to the prevalence of SLE in the region [1], this correlation must be investigated and elucidated.

\section{Aim and objective}

Given the deficiency of solid evidence of this correlation in the UAE, we aimed, in a nested case-control study, to investigate whether children born to women with SLE have an increased risk of CHD when compared to children born to women without SLE. This study will help us address the gap in the literature, as well as, help in facilitating better management of both SLE and CHDby promoting better patient education with regards to the risks of SLE on pregnancies with evidence based medicine. Furthermore, results of this study will encourage superior resource allocation with regards to the treatment and management of SLE and CHD.

\section{Hypothesis}

The key research question of this study is whether or not SLE serves as a risk factor for developing CHD in the children born to the women with this disorder. We hypothesize that there is a positive correlation between SLE and CHD in which a child born to a mother with SLE would be at a higher risk of having CHD when compared to children born to non-SLE mothers.

\section{Methods}

This study is a case-control study nested in a retrospective cohort in which we selected the desired study sample from a population of pregnant women between 2015 and 2017 from a hospital in Dubai. Put simply, we gathered women with the desired exposure from a cohort and we compared them with other women without the exposure, from the same cohort, to see whether or not they were more likely to develop the outcome of interest. Patient records used were from Al Jalila Children's Hospital (AJCH) where women who gave birth were followed up. The records contained all the information needed regarding the health status of both the child and the mother, including and not limited to, the mother's past history and the in utero development notes of the fetus. The 2015 to 2017 timeframe was selected as it is the farthest date available, with regards to patient records. This is accounted for by the recent launch of the hospital in 2015. Patients who satisfied the inclusion criteria were recruited from that respective sample.

\section{Ethical obligations}

I have, and to the fullest of extents, followed the international ethical obligations. I have maintained full patient's confidentiality when reviewing patients records and protected their privacy. Wherever used in my research, patient's names and identifiers were coded and shuffled. My research proposal had been approved by the Mohammed Bin Rashid University - Institutional Review Board (MBRU-IRB-SRP2018-045) and by Al Jalila Children's Hospital Director of Research Department.

\section{Eligibility criteria}

The inclusion criteria for the exposed group were women in AJCH aged between 20-40 who have been clinically diagnosed with SLE previously and who were pregnant. The diagnosis of SLE was based on positive Anti-Ro/Anti-La antibodies in serum blood tests along with the presence of identifying diagnostic clinical features. On the other hand, the inclusion criteria for the controls was similar in terms of age however they did not have SLE or any other chronic illnesses on record. Women who were younger than 20 years old or older than 40 years old were eliminated from the study. In both groups, any woman with a history of hypertension, diabetes or any other chronic ailments were removed from the study. Women who were smokers were not included into the study. Twin pregnancies were also eliminated from the study.

\section{Outcome assessment}

The outcome of interest was the presence of CHD in the fetus, this was defined as any structural and/or conductive abnormality seen in the heart on the fetal echocardiogram. We did not define any specific heart abnormalities that we were looking for, and included any heart abnormality seen. The echocardiograms were carried out by one doctor to rule in/out the presence of any abnormalities. They were not scheduled fetal echocardiograms and they took place anytime during the gestation period. However, in some abnormal findings, a second echocardiogram was required a few weeks after the initial scan to see if the heart had recovered or not. The final echocardiogram was considered; any previous echo scan was disregarded.

\section{Assessing relevant covariates}

For all mothersin our study, we reviewed their records to identify any pre-existing and current co-morbidities (i.e. hypertension, asthma, diabetes) recorded. Covariates such as nationality, age of mother and age of fetus at time of echocardiogram were taken into account in the statistical analysis to rule out any significance to the main correlation in the study. Mother's medications usage was disregarded and not included into the statistical analysis. 


\section{Bias}

We are aware of the potential biases that could have impacted the results of our study. However, to account for recall bias, our study relied on previously recorded information, which minimizes the chance of recording exaggerated exposures. Moreover, observer bias was eliminated as the exposure was listed in the patient record and was not measured for this study only. To account for selection bias, we selected cases and controls from the same registry with similar variables with the only difference being the presence of the exposure (SLE) or not. We also recruited more controls than cases (2:1 ratio, respectively) to help us improve the statistical power of the study. Furthermore, there is the risk of detection bias. Children of chronically ill mothers are more likely to undergo fetal echocardiography as part of routine screening to detect CHD in those exposed to in utero Anti-Ro/La which are present in SLE women. This means CHD may be more easily detected in children born to women with SLE than in controls, leading to an overestimation of the association.

\section{Statistical Analysis}

We used the IBM Statistical Package for the Social Science (SPSS) Version 2413 to calculate the prevalence of CHD in both groups of cases and controls. Following that, we calculated the odds ratio (OR) for developing CHD in both the exposed and unexposed groups. We then carried out a multivariable logistical regression test for age of fetus, age of mother and nationality. Of note, we excluded exposure to medications as the records were incomplete and missing information.

\section{Result}

From the Chi-Squared tables (Table 1), we could see a total of 122 women were inducted into the study; of which, 36 (29.5\% of total) women had SLE compared to 86 (70.5\% of total) women without SLE (1:2 ratio, respectively). In the SLE exposed group, 5 (13.8\%) women had children with CHD, compared to 35 women in the non-SLE group (40\%) Mean maternal age in the overall sample of mothers was 32.6 (standard deviation, SD, 4.9). When carrying out a risk estimate (Table 2), we could see that the SLE group (OR 4.2, 95\%CI 0.083-0.664) has a higher risk of having a child with CHD when compared to the non-SLE group. Women with SLE are 4.2 times more likely to have a child with CHD than women who do not have SLE. This result was also confirmed on Fisher's exact test (2-sided) which gave a P-value of 0.005 showing significant difference in the two groups. In logistical regression (Table 3) including all the women, we tested for age of fetus, age of mother and nationality to explore the relationship between the dependent variable and the independent variables. We observed here that none of these variables play a significant role in influencing the main correlation (Table 1-3)

Table 1: Chi-Squared Cross-tabulations displaying the frequency of congenital heart defects in relation to exposure to SLE in Al Jalila Children's Hospital patient records, Dubai, 2015-2017.

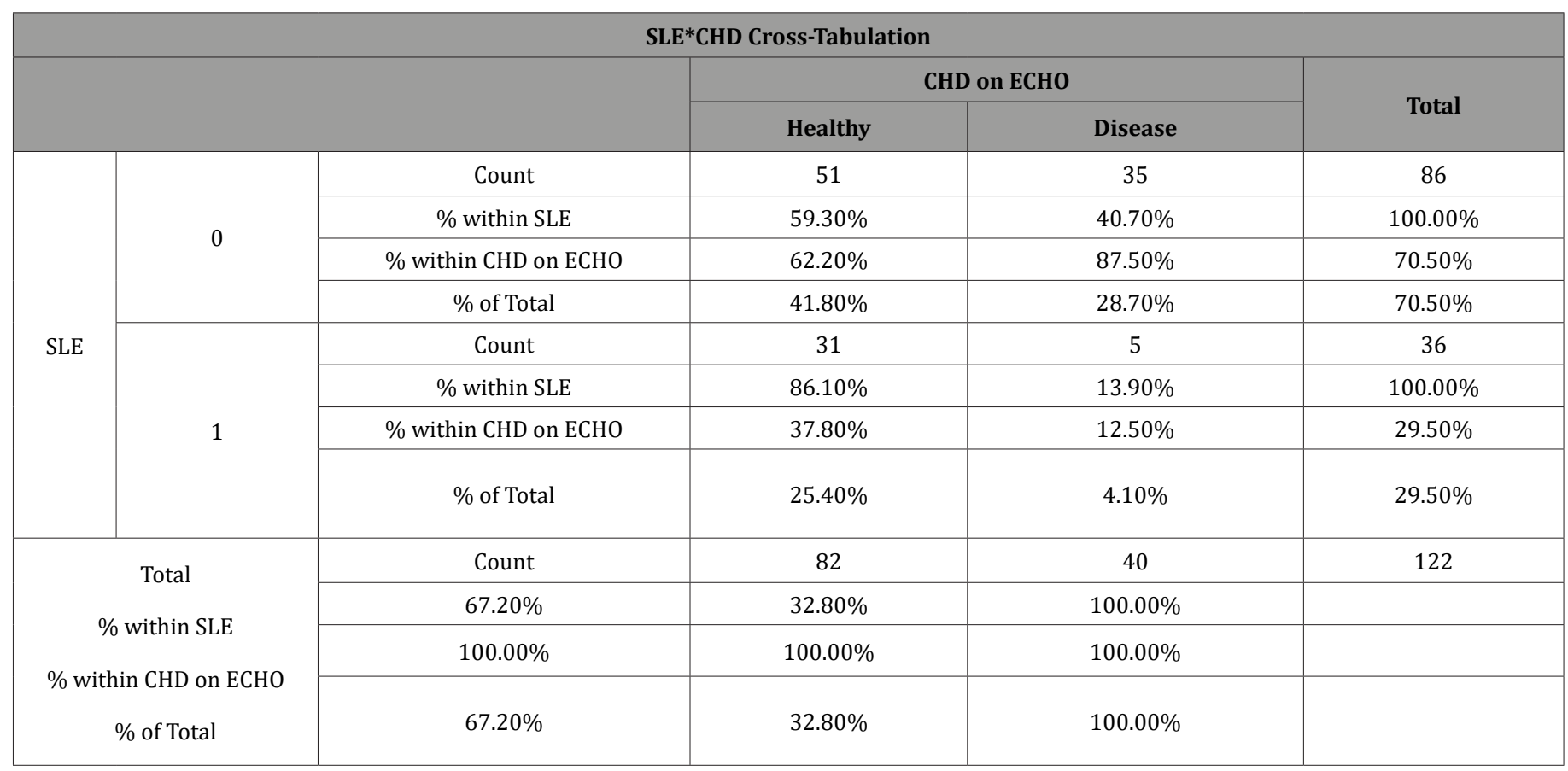

SLE - Systemic lupus erythematosus

CHD - Congenital Heart Defects

\section{Discussion}

\section{Major findings}

We found an association between SLE and CHD in which, children who are born to mothers with SLE have a higher risk of developing CHD when compared to children born to women without SLE. This is true as a 2014 study in Quebec, Canada found this same correlation [4]. The Offspring of SLE mother Registry (OSLER) was a large cohort study looking at patient records in the Quebec province between 1989 and 2009, the study included 6,333 women involved in the study (509 cases and 5824 matched controls, 4:1 controls to cases ratio) which showed that children born to SLE women have a higher risk of CHD compared to non- 
SLE women. The results from our study help us in accepting our research hypotheses and rejecting the null hypothesis; Also they are in par with the published literature in terms of correlation.

When carrying out the risk assessment, we found that the odds of developing CHD if the child is exposed to SLE are 4.2 times higher in the exposed when compared to the non-exposed group. We can see it by calculating the reciprocal of the value indicated in Table 2 $(1 / 0.235=4.2)$. The odds ratio came with a $95 \%$ CI between 0.083 and 0.664 . From the confidence interval value obtained, we can say that we are $95 \%$ confident that the odds ratio of developing CHD in a child exposed to maternal SLE in utero is 4.2 times more likely than in the unexposed group, since the $95 \% \mathrm{CI}$ does not include the null value, thus this finding is statistically significant.

Table 2: Risk analysis highlighting the Odds Ratio of developing CHD in non-SLE group to SLE group. Reciprocal of this value was used.

\begin{tabular}{|c|c|c|c|}
\hline \multicolumn{4}{|c|}{ Risk Estimate } \\
\hline & Value & \multicolumn{2}{|c|}{$\mathbf{9 5 \% \text { Confidence Interval }}$} \\
\hline $\begin{array}{c}\text { Odds Ratio for } \\
\text { SLE (0/1) }\end{array}$ & 0.235 & 0.083 & 0.664 \\
\hline $\begin{array}{c}\text { For cohort } \\
\text { CHD on } \\
\text { ECHO=healthy }\end{array}$ & 0.689 & 0.553 & 0.857 \\
\hline $\begin{array}{c}\text { For cohort } \\
\text { CHD on } \\
\text { ECHO=disease }\end{array}$ & 2.93 & 1.249 & 6.873 \\
\hline N of Valid Cases & 122 & & \\
\hline
\end{tabular}

In multivariable logistic regression, we could observe that the three variables we tested for (age of fetus, age of mother and nationality) play no part in predicting the risk of developing CHD in and SLE exposed child. We can see from Table 3 that the three variables each come with a level of significance, and based on the cut off of 0.05 , none of these are of any relevant significance. This is also confirmed through the $95 \% \mathrm{CI}$, which includes the null value, indicating non-significant statistical findings.

Table 3: Logistical regression analysis involving multi-variables to assess their influence on the main correlation of this study.

\begin{tabular}{|c|c|c|c|c|c|c|}
\hline \multicolumn{7}{|c|}{ Logistical Regression } \\
\hline & B & Sig & \multirow{2}{*}{$\operatorname{Exp(B)}$} & \multicolumn{2}{|c|}{$95 \%$ CI for EXP(B) } \\
\cline { 5 - 8 } & & & Lower & Upper \\
\hline \multirow{5}{*}{ Step 1a } & $\begin{array}{c}\text { Age of } \\
\text { Fetus }\end{array}$ & -0.001 & 0.984 & 0.999 & 0.913 & 1.093 \\
\cline { 2 - 7 } & Age & -0.07 & 0.093 & 0.932 & 0.859 & 1.012 \\
\cline { 2 - 7 } & Nationality & -0.629 & 0.124 & 0.533 & 0.239 & 1.189 \\
\cline { 2 - 7 } & SLE & -1.435 & $.008^{*}$ & 0.238 & 0.082 & 0.69 \\
\cline { 2 - 7 } & Constant & 2.859 & 0.139 & 17.451 & & \\
\hline
\end{tabular}

*-significance less than 0.05 (cut off).

\section{Clinical correlation}

The pathophysiology behind SLE's influence on the developing heart is multifactorial and there is no factor we could point out as the main culprit. Mechanisms including autoantibody-mediated damage and cytokine imbalance are thought to be the causative factors in the deformation of the fetal heart. IgG antibodies are notoriously known for their ability to cross the placenta and exert their damage on the fetus, such as in Rhesus Babies [5,6].

Anti-SSA/Ro and Anti-SSB/La are found in around 40\% of women with SLE and are IgG antibodies. They have the ability to cross the placenta and they do so via active transport, making their levels very high in the fetus. They are associated with the development of neonatal lupus which has a cardiac manifestation of congenital heart block in utero. It has been demonstrated by investigators that these Anti-Ro/Anti-La bind to the fetal cardiocytes and result in the release of pro-inflammatory and pro-fibrosing cytokines and eventually causing scarring [7]. These pathways are thought to be the cause of the congenital heart block seen in the fetus at that stage, however an investigation of autopsies of cardiac neonatal lupus cases revealed, histologically, that the damage was beyond the conduction system and involved the endocardium, myocardium and valves [8]. Thus, damage is not limited to the conduction system of the heart, but to a greater extent, the entire heart.

It is known that the development of the cardiac septum occurs by the 6th week of gestation and since the maternal antibodies only start crossing the placenta at 20 weeks of gestation, it is unlikely that the presence of those antibodies would be the cause of defects in the ventricular septum. It has been investigated that the defect in the ventricular septum (VSD) would occur due to a process of active remodelling of an already developed septum rather than from the early formation [9]. This is, in addition to, the theory that the maternal antibodies might prevent the closure of a defect in the septum that might've been closed otherwise which could possibly explain the risk of CHD in children born to SLE mothers.

Transforming Growth Factor beta (TGF-B) is a cytokine that plays a critical role in cardiac embryogenesis during the formation of the endocardium [10]. On mice studies, it was found that mice with no TGF-B born to mothers without TGF-B had severe cardiac complications. When the mouse missing TGF-B was born to a mother with TGF-B, it was found that the offspring had less severe CHD10. What is intriguing is that women with SLE have lower levels of TGF-B than the general population [11]. This could mean that maternal TGF-B could salvage the fetal defective heart in non-SLE mother; on the other hand, in SLE mothers and due to the low levels of maternal TGF-B, this restoration of the fetal heart may be absent.

\section{Limitations}

Mothers with chronic illnesses are more likely to have more frequent follow ups and scan during their pregnancy period when compared to women with no chronic illnesses. SLE mothers are more concerned about their fetus's health than non-SLE mothers and so they will seek medical attention more frequently. Since we are using fetal echocardiograms to detect the outcome of interest, SLE mothers may have more than one fetal echocardiogram which could lead to an overestimation of the cases of CHD of the children born to these mothers. This detection bias came from the limited information in the patient records and could not be removed from our study and thus it serves as a limiting factor. Supposing that we had more detailed look over the patient records, we would have eliminated those mothers with $>1$ fetal echocardiogram from the study. 
Furthermore, and due to lacking patient records, we could not factor in the medications used by the mother during her pregnancy. Information regarding the mother's medications were poorly recorded into the files and there were many contrarieties. These medications would include corticosteroids and anti-malarial medications which are used to reduce SLE flares during pregnancy. The OSLER study found an association between exposure to corticosteroids in utero and CHD [4], however the results had a wide confidence interval and other publications are uncertain of this association.

The patient records also had lacked information regarding smoking and alcohol intake of those mothers enrolled in the study. This serves as a limiting factor as those variables could heavily influence the correlation being studied.

\section{Areas for future research}

This research could be further expanded to cover more variables such as the effects of SLE medications on the developing heart. The study could include more hospitals around the U.A.E and seek more complete patient records. Also, we could specify which types for CHD were forming instead of using an umbrella term. This would enable us to pinpoint which is most likely to form due to SLE exposure. The aforementioned limitations and biases encountered could be avoided in the future. Other studies could also investigate and rule out the effects of other in utero exposures on the developing heart.

The effects of diabetic embryopathy could have been investigated by collecting data on the pregestational and gestational diabetes of those mothers. It is known that major congenital abnormalities are seen in women with poorly controlled pregestational diabetes and at lower severity in gestational diabetes [12]. The data about gestational diabetes was not recorded in the patient records and thus this association could not be tested [13].

In addition to that, from the fetal echocardiogram reports, we could have reported which types of CHD were seen in the fetus (ventricular septal defects, atrial septal defects etc.) which would help us see which type of CHD is most prevalent and most likely to form.

As the data from this study were collected from a single hospital in Dubai and the small sample size, play a role in the external validity and generalizability of the results.

\section{Conclusion}

In conclusion, our study has found that children born to mothers with SLE have a higher risk of developing CHD when compared to children born to mothers without SLE. These findings are in par with the literature being reported from around the globe and thus it is externally valid. This study prompts further investigations to elucidate the role of risk-factors, such as in utero drug exposures and cytokines, in explaining the increased likelihood of developing CHD in the children born to mothers with SLE. This study can help promoting better patient care by providing evidence based medicine advice to those mothers with SLE attempting to start a family.

\section{Acknowledgement}

Special thanks to Dr. Mahmoud AlSoufi (AJCH) for his continuous support and motivation throughout the course of this study and to Dr. Amar Hassan for his help in the statistical analysis.

\section{Conflict of Interest}

No conflict of interest.

\section{References}

1. Dhanhani AAl, Bakoush O, Agarwal M, Othman Y (2017) The Incidence and Prevalence of Systemic Lupus Erythematous in a Defined Population in United Arab Emirates. Arthritis Rheumatol 26(6): 664-669.

2. Al Mazrouei SK, Moore J, Ahmed F, Mikula EB, Martin GR, et al. (2013) Regional Implementation of Newborn Screening for Critical Congenital Heart Disease Screening in Abu Dhabi. Pediatr Cardiol 34: 1299-1306.

3. Liu S, Joseph KS, Lisonkova S, Rouleau J, Van den Hof M, et al. (2013) Association Between Maternal Chronic Conditions and Congenital Heart Defects. Circulation 128: 583-589.

4. Vinet É, Pineau CA, Scott S, Clarke AE, Platt RW, et al. (2015 ) Increased congenital heart defects in children born to women with systemic lupus erythematosus results from the offspring of systemic lupus erythematosus mothers registry study. Circulation 131(2): 149-56.

5. Costumbrado J, Ghassemzadeh SRh (2018) Incompatibility. StatPearls.

6. Garty BZ, Ludomirsky A, Danon YL, Peter JB, Douglas SD, et al. (1994) Placental transfer of immunoglobulin G subclasses. Clin Diagn Lab Immunol 1(6): 667-669.

7. Izmirly PM, Buyon JP, Saxena A (2012) Neonatal lupus: advances in understanding pathogenesis and identifying treatments of cardiac disease. Curr Opin Rheumatol 24: 466-472.

8. Llanos C, Friedman DM, Saxena A, Izmirly PM, Tseng CE, et al. (2012) Anatomical and pathological findings in hearts from fetuses and infants with cardiac manifestations of neonatal lupus. Rheumatology 51: 1086092 .

9. Kenzo Ivanovitch, Isaac Esteban, Miguel Torres (2017) Growth and Morphogenesis during Early Heart Development in Amniotes. J Cardiovasc Dev Dis 4(4): 20.

10. Arthur HM, Bamforth SD (2011) TGF $\beta$ signaling and congenital heart disease: Insights from mouse studies. Birth Defects Res Part A Clin Mol Teratol 91: 423-434.

11. Su DL, Lu ZM, Shen MN, Li X, Sun LY, et al. (2012) Roles of Pro- and AntiInflammatory Cytokines in the Pathogenesis of SLE. J Biomed Biotechnol 2012: 1-15

12. Corrigan N, Brazil DP, McAuliffe F (2009) Fetal cardiac effects of maternal hyperglycemia during pregnancy. Birth Defects Res Part A Clin Mol Teratol 85: 523-530.

13. IBM Corp (2016) IBM SPSS Statistics for Windows, Version 24.0. Armonk, NY: IBM Corp. 